\title{
Choreoathetosis and dystonia in a child with COVID-19 and multisystem inflammatory syndrome
}

\author{
P Z Mteshana, ${ }^{1}$ FCPaed (SA); K D Naidoo, ${ }^{2}$ Cert Critical Care; V Rowjee, ${ }^{2}$ FCPaed (SA); \\ M P K Hauptfleisch, ${ }^{1}$ Cert Paed Neuro (SA); Z Dangor, ${ }^{1}$ Cert Paed Pulmonology \\ ${ }^{1}$ Department of Paediatrics and Child Heath, Chris Hani Baragwanath Academic Hospital, University of the Witwatersrand, Johannesburg, \\ South Africa \\ ${ }^{2}$ Paediatric Critical Care, Department of Critical Care, Chris Hani Baragwanath Academic Hospital, University of the Witwatersrand, \\ Johannesburg, South Africa.
}

\section{Corresponding author: P Mteshana (Phindile.Mteshana@wits.ac.za}

\begin{abstract}
Neurological complications of COVID-19 or multisystem inflammatory syndrome in children (MIS-C) are well described. We report an unusual presentation in a 9-year-old girl presenting with status epilepticus, who thereafter developed choreoathetosis and dystonia. She was initially managed with intravenous immunoglobulins and methylprednisolone for presumed autoimmune encephalitis. However, she tested positive for SARS-CoV-2 and met the clinical and laboratory criteria for MIS-C. She remained encephalopathic with abnormal movements and dystonia for 8 days from presentation but was discharged home with complete clinical recovery after 2 weeks.
\end{abstract}

S Afr J Child Health 2021;15(4):229-231. https://doi.org/10.7196/SAJCH.2021.v15i4.1859

The novel coronavirus (SARS-CoV-2) was first reported in December 2019 in Wuhan, China, and has become a global pandemic. ${ }^{[1]}$ The first reported case in South Africa (SA) was on 5 March 2020. ${ }^{[2]}$ In late April 2020, an alert about the multisystem inflammatory syndrome in children (MIS-C) and adolescents between the ages of 0 and 19 years was issued. ${ }^{[3]}$ This syndrome is characterised by fever, inflammation and evidence of multi-organ involvement. ${ }^{[3]}$ Case definitions have been proposed by the World Health Organization (WHO), the Royal College of Paediatrics and Child Health and the Centers for Disease Control and Prevention - most of the clinical and laboratory criteria are similar. ${ }^{[3-6]}$ The WHO classification system has been adopted in SA.

Coronavirus disease 2019 (COVID-19) can present with a range of neurological manifestations including headache, decreased level of consciousness, seizures, encephalopathy and disturbance of smell and taste, as reported in adult patients. ${ }^{[1]}$ An adult study identified five categories of neurological presentations:(i) encephalopathies with delirium/psychosis and no distinct magnetic resonance imaging (MRI) or cerebrospinal fluid (CSF) abnormalities; (ii) inflammatory central nervous system syndromes including encephalitis, myelitis and acute disseminated encephalomyelitis; (iii) ischaemic strokes associated with a prothrombotic state; (iv) peripheral neurological disorders; and $(v)$ miscellaneous central disorders. In addition, neurological organ involvement has been described in some case definitions of MIS-C and encompasses seizures, coma and encephalitis, among others. ${ }^{[7]}$ This case report describes an unusual neurological presentation in a child with COVID-19 who met the criteria for MIS-C.

\section{Case presentation}

A 9-year-old HIV-negative girl presented to the Chris Hani Baragwanath Academic Hospital during the first wave of COVID-19. She had a fever and was in status epilepticus. She reported a preceding 3-day history of headache, multiple episodes of vomiting, rhinorrhoea, cough and fever. Her temperature on arrival was $38.3^{\circ} \mathrm{C}$, she had a normal blood glucose level and she displayed no features of hypoperfusion. On clinical examination, she was encephalopathic but with normal tone and reflexes. She needed two doses of intravenous midazolam and two phenytoin loading doses $(20+10 \mathrm{mg} / \mathrm{kg}$ intravenously) to abort the seizures. A lumbar puncture was initially deferred, as she had a reduced level of consciousness (Glascow Coma Scale (GCS) 10: E4M4V2), and intravenous ceftriaxone was commenced empirically to cover for possible bacterial meningitis. The blood work on admission showed leukocytosis with neutrophilia and lymphopenia, and normal C-reactive protein (CRP) (Table 1). The computed tomography scan of the brain (CTB) was unremarkable.

Her temperature remained above $38.5^{\circ} \mathrm{C}$ for the first 24 hours of admission. On the second day of admission, she developed dystonia, choreoathetosis and facial grimacing with hypotonia and normal reflexes. The working diagnosis was autoimmune or viral encephalitis. Phenytoin-induced dyskinesia was also considered, given that two loading doses of phenytoin were needed to abort her seizures. She was treated with intravenous immunoglobulin ( $2 \mathrm{~g} / \mathrm{kg} / \mathrm{day}$, administered over 2 days), methylprednisolone (30 mg/kg/day, for 5 days) followed by oral prednisolone $(1 \mathrm{mg} / \mathrm{kg} /$ day), and acyclovir was commenced on day 2 of her hospitalisation. The CSF was acellular with normal biochemistry and without organism growth on culture. The CSF and blood were sent for testing for oligoclonal bands and N-methyl-D-aspartate receptor (NMDAR) antibodies. There was insufficient CSF for viral PCR studies. Common toxins were screened for and excluded.

Within 48 hours of admission, the SARS-CoV-2 nasopharyngeal swab taken on admission was confirmed as positive, and she was transferred to the paediatric COVID-19 isolation ward. The hospital did not have access to SARS-CoV-2 antibody testing at the time of her presentation. On day 4, her CRP increased to $257 \mathrm{mg} / \mathrm{L}$ and her extrapyramidal signs persisted. Her clinical presentation and 
laboratory results were compatible with a diagnosis of MIS-C; she had fever, evidence of SARS-CoV-2, organ involvement, nonpurulent conjunctivitis, coagulopathy and raised inflammatory markers (Table 1). Low-molecular-weight heparin was added to the treatment because of raised serum d-dimers, and azathioprine was initiated because of the persistence of encephalopathy and abnormal movements.

On the 10th day of admission, she began to show signs of improvement. The abnormal movements became less apparent and GCS normalised. By the 14th day, she was ambulating independently, and able to feed herself and communicate. The repeat SARS-CoV-2 swab was negative on the 15th day, and she was discharged home the following day.

\section{Ethical considerations}

Consent for the writing of this report was obtained from the parents. Ethics approval was obtained from the University of the
Witwatersrand Human Research Ethics Committee (HREC ref. no. M2009101).

\section{Discussion}

To our knowledge, this is the first reported clinical presentation of choreoathetosis and dystonia in a child with COVID-19. It remains unclear whether the unusual clinical presentation in this case was a manifestation of COVID-19 itself or part of MIS-C. There have been other reported cases of neurological involvement associated with COVID-19 and MIS-C in children, but none has described choreoathetosis and dystonia. ${ }^{[7-9]}$ The proposed pathogenesis of neurological involvement includes infection of neurological tissue, maladaptive inflammatory and/or immune-mediated host responses, vascular endothelial injury or cerebrovascular disease caused by coagulopathy. ${ }^{[10,11]}$ Another possibility was phenytoin-induced dyskinesia, which may occur in cases with toxic serum levels, polypharmacy or underlying neurological

Table 1. Laboratory investigation undertaken in this child

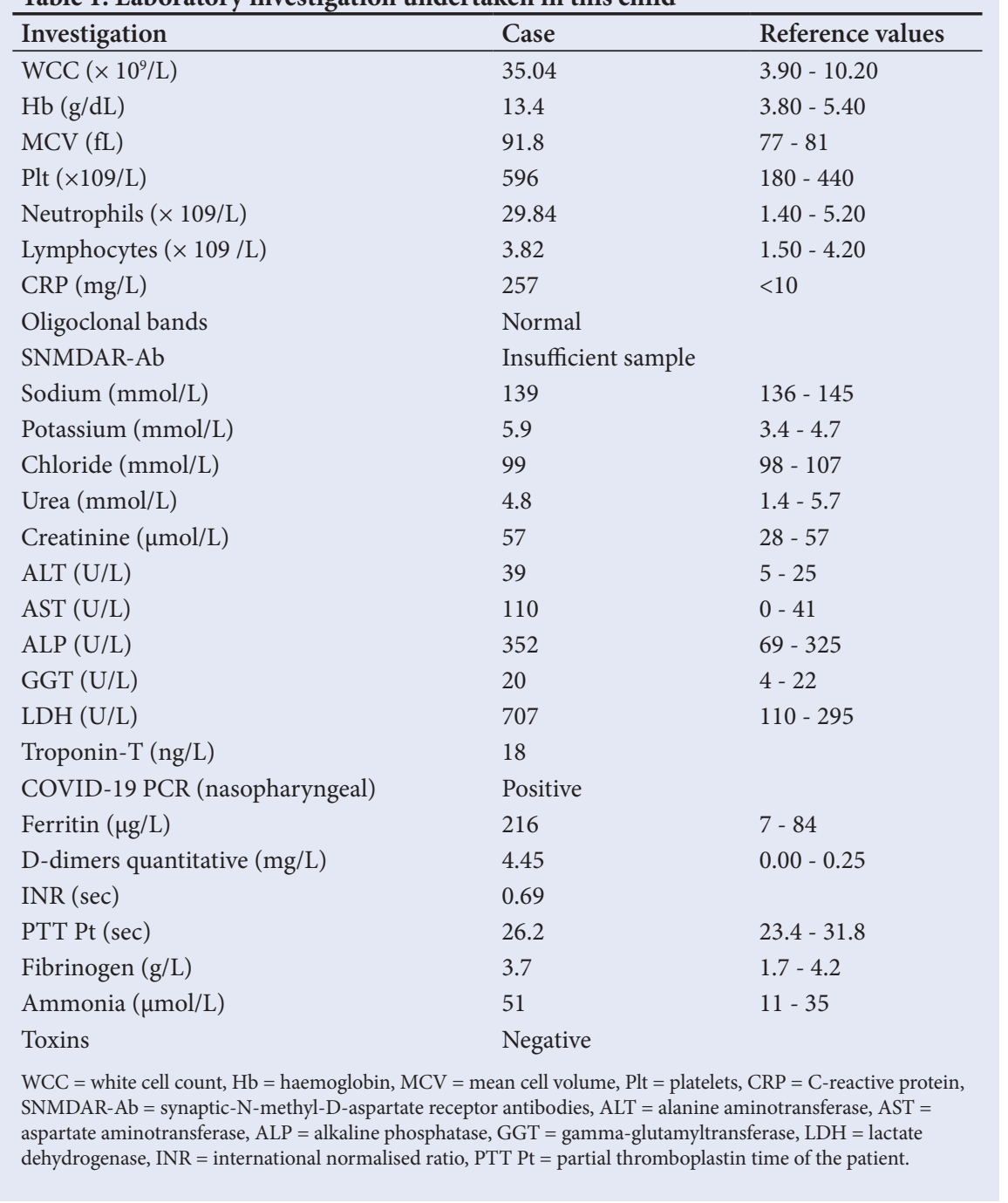

diseases; however, it does not generally last longer than the serum half-life. ${ }^{[12,13]}$ This diagnosis was considered less likely because the choreoathetosis and dystonia manifested 17 hours after phenytoin was administered, and persisted for a further 8 days.

Case reports of two children with severe neurological complications associated with MIS-C described middle cerebral artery infarction in both patients. One of the children had cerebral oedema and diffuse subarachnoid haemorrhage, and the other had a posterior cerebral artery infarct. ${ }^{[14]}$ In another case series of 27 children with MIS-C, 4 had neurological findings such as encephalopathy, headaches, brainstem and cerebellar signs, muscle weakness and reduced reflexes. ${ }^{[8]}$

While CTB remains an excellent diagnostic tool for patients with infarction and/or haemorrhage, ${ }^{[14]}$ it appears less sensitive in patients with other neurological complications associated with COVID-19, particularly encephalitis. $^{[8,9,15,16]}$ Our patient's CTB was reported to be normal. In contrast, MRI has proved much more useful both in children and adults with COVID-19. ${ }^{[17]}$ All four of the abovementioned children with MIS-C and neurological involvement demonstrated possibly reversible lesions in the splenium of the corpus callosum from inflammationinduced focal intramyelin oedema. ${ }^{[8]}$

Electroencephalography (EEG) is another useful investigation in encephalopathic patients with COVID-19, showing an excess of slow activity even when MRI has failed to detect abnormalities. ${ }^{[8,15]}$ Given these initial data, both MRI and EEG are essential diagnostic investigations in children with neurological signs associated with COVID-19 or MIS-C. Our patient, however, did not undergo these investigations for fear of contamination of the single MRI suite and limited portable EEG machines in our setting. Similarly, in other low-resourced settings, children with COVID-19 have been denied access to important and necessary investigations. ${ }^{[18]}$

The management of COVID-19-associated neurological disorders remains the subject of debate and scientific enquiry. Some have opted to manage these patients with no specific therapy and have reported full recovery, ${ }^{[9]}$ while others have used agents such as mannitol to decrease intracerebral pressure and provide symptomatic relief. ${ }^{[16,19]}$ In our setting, the South African Paediatric Critical Care Working Group recommends a combination of intravenousimmunoglobulins and systemic glucocorticoids together with aspirin in patients with MIS-C. ${ }^{[20]}$ Similarly, three of the four children mentioned above 
were treated with immunomodulatory agents (methylprednisolone, dexamethasone, intravenous immunoglobulin, anakinra and rituximab) $\cdot{ }^{[8]}$

\section{Conclusion}

This unique case presentation of choreoathetosis and dystonia highlights additional neurological signs in children with COVID-19 and/or MIS-C. Furthermore, the road to recovery in this child was favourable despite the initial poor response to therapy - this warrants further investigation.

Acknowledgements. We thank the team that looked after this patient during her hospital stay, our patient and her parents for allowing us to write up this case.

Author contributions. PZM did the initial write-up. MPKH, VR, KDN and ZD provided clinical input and oversight. All authors approved the final draft.

Funding. None.

Conflicts of interest. None.

1. Guan WJ, Ni ZY, Hu Y, et al. Clinical characteristics of coronavirus disease 2019 in China. N Engl J Med 2020;382(18):1708-1720. https://doi.org/10.1056/ NEJMoa2002032

2. About COVID-19 (Coronavirus). https://sacoronavirus.co.za/informationabout-the-virus-2/ (accessed 21 April 2020)

3. World Health Organization. Multisystem inflammatory syndrome in children and adolescents with COVID-19 2020. https://www.who.int/publications/i/ item/multisystem-inflammatory-syndrome-in-children-and-adolescentswith-COVID-19 (accessed 15 May 2020).

4. Centers for Disease Control and Prevention. Multisystem inflammatory syndrome in children (MIS-C) associated with coronavirus disease 2019 (COVID-19) 2020. https://emergency.cdc.gov/han/2020/han00432.asp (accessed 14 May 2020).

5. Royal College of Paediatrics and Child Health. Guidance - Paediatric multisystem inflammatory syndrome temporally associated with COVID-19 (PIMS) 2020. https://www.rcpch.ac.uk/resources/guidance-paediatricmultisystem-inflammatory-syndrome-temporally-associated-COVID-19pims. (accessed 1 May 2020)
6. National Institute of Communicable Diseases. MIS-C is now a notifiable condition in South Africa. https://www.nicd.ac.za/mis-c-is-now-a-notifiablecondition-in-south-africa/ (accessed 18 September 2020 ).

7. Feldstein LR, Rose EB, Horwitz SM, et al. Multisystem inflammatory syndrome in U.S. children and adolescents. N Engl J Med 2020;383(4):334346. https://doi.org/NEJMoa2021680

8. Abdel-Mannan $\mathrm{O}$, Eyre $\mathrm{M}$, Löbel $\mathrm{U}$, et al. Neurologic and radiographic findings associated with COVID-19 infection in children. JAMA Neurol 2020;77(11):1440-1445. https://doi.org/10.1001/jamaneurol.2020.2687

9. McAbee GN, Brosgol Y, Pavlakis S, Agha R, Gaffoor M. Encephalitis associated with COVID-19 infection in an 11-year-old child. Pediatr Neurol 2020;109:94. https://doi.org/10.1016\%2Fj.pediatrneurol.2020.04.013

10. Ellul MA, Benjamin L, Singh B, et al. Neurological associations of COVID-19. Lancet Neurol 2020;19(9):767-783. https://doi.org/10.1016/ s1474-4422(20)30221-0

11. Lin JE, Asfour A, Sewell TB, et al. Neurological issues in children with COVID-19. Neurosci Lett 2021;743:135567. https://doi.org/10.1016/j. neulet.2020.135567.

12. Filloux F, Thompson JA. Transient chorea induced by phenytoin, J Pediatr 1987;110(4):639-664. https://doi.org/10.1016/S0022-3476(87)80570-X

13. Craig S. Phenytoin poisoning. Neurocrit Care 2005;3:161-170. https://doi org/10.1385/ncc:3:2:161

14. Schupper AJ, Yaeger KA, Morgenstern PF. Neurological manifestations of pediatric multi-system inflammatory syndrome potentially associated with COVID-19. Childs Nerv Syst 2020;36(8):1579-1580. https://doi.org/10.1007/ s00381-020-04755-8

15. Pilotto A, Odolini S, Masciocchi S, et al. Steroid-responsive encephalitis in coronavirus disease 2019. Ann Neurol 2020;88(2):423-427. https://doi. org/10.1002/ana.25783

16. Ye M, Ren Y, Lv T. Encephalitis as a clinical manifestation of COVID-19. Brain Behav Immun 2020;88:945-946. https://doi.org/10.1016/j.bbi.2020.04.017

17. Aghagoli G, Gallo Marin B, Katchur NJ, Chaves-Sell F, Asaad WF, Murphy SA. Neurological involvement in COVID-19 and potential mechanisms: A review. Neurocrit Care 2020:1-10. https://doi.org/10.1007\%2Fs12028-020-01049-4

18. Mao $L$, Jin $H$, Wang $M$, et al. Neurologic manifestations of hospitalised patients with coronavirus disease 2019 in Wuhan, China. JAMA Neurol 2020;77(6):1-9. https://doi.org/10.1001/jamaneurol.2020.1127

19. Shi Y, Wang X, Liu G, et al. A quickly, effectively screening process of novel corona virus disease 2019 (COVID-19) in children in Shanghai, China. Ann Trans Med 2020;8(5):241. https://doi.org/10.21037/atm.2020.03.22

20. Rossouw B, McCulloch M. Paediatric critical care during the COVID-19 pandemic. S Afr J Crit Care 2020;36(1):10-11. https://doi.org/10.7196/ SAJCC.2020.v36i1.452

Accepted 16 March 2021. 